Rev. Elev. Méd. vét. Pays trop., 1973, $26(2) \cdot 245-48$

\title{
Influence de l'âge à la castration sur le développement et le rendement du zébu à Madagascar
}

\author{
par J. GILIBERT, P. DUBOIS (*)
}

\begin{abstract}
RESUME
A Madagascar, la quasi-totalité des éleveurs pratique un élevage extensif non supplémenté pour la production de viande avec des animaux de race Zébu malgache.

La castration des animaux est effectuée entre 2 et 4 ans d’âge. Les auteurs ont étudié l'influence de l'âge à la castration sur le développement ultérieur, en castrant des animaux à 8 mois, 12 mois et 24 mois; tous les animaux ont été abattus à quatre ans et demi après avoir été ćlevés selon le mode traditionnel. Dans cette expérience, l'abaissement de l'âge de la castration augmente la croissance pondérale globale, n'a pas d'influence sur le rendement en carcasse et augmente le rapport largeur sur longueur du bassin.
\end{abstract}

A Madagascar en élevage extensif traditionnel, la castration est pratiquée sur la quasitotalité des animaux mâles, mais à un âge avancé ( $3 \pm 1$ an). Si quelques arguments sont émis en faveur de cette castration tardive croît plus rapide des mâles, choix des taureaux et meilleure résistance aux conditions défavorables de la saison sèche, notamment - la raison essentielle est dans son caractère coutumier, alors qu'elle est unanimement prônée pour l'obtention de bœufs de travail.

Une minorité d'éleveurs pratique la castration de leurs taurillons à un âge plus jeune, vers 30 mois environ, avec satisfaction. Il s'agit là d'une coutume appelée «Vositra-Kely qui signifie : jeune castré (bouvillon).

Notre étude a eu pour objet principal de préciser l'influence que peut présenter le facteur « âge à la castration » pour l'obtention de bœufs de boucherie d'herbe, élevés selon le mode d'élevage extensif traditionnel.

(*) Région de Recherches Vétérinaires et Zootechniques de Madagascar, B.P. no 862, Tananarive, République Malgache.

\section{MATERIEL ET METHODES}

Les animaux concernés sont des Zébus de race malgache nés au Centre de Recherches Zootechniques de Miadana dans des troupeaux n'ayant jamais été l'objet de sélection. A l'exception d'une période de 3 mois consécutive au sevrage, les animaux sont élevés sur pâturage naturel non supplémenté. La saison sèche dure entre 6 et 7 mois selon les années. La pratique d'une saison de monte permet de grouper les naissances en quatre mois.

Deux séries de comparaisons ont été effectuées: la série $\mathrm{A}$ concerne des animaux nés en 1966, dont un lot a été castré à 12 mois (A 12), un autre à 18 mois (A 18); de plus, quatre taurillons qui avaient été présélectionnés pour la reproduction, ont été castrés à l'âge de 24 mois et incorporés à l'expérience (A 24).

La série B concerne des animaux nés en 1967 , dont un lot a été castré à 8 mois (B 8) et l'autre à 12 mois (B 12). Tous les animaux ont été castrés avec la pince de Burdizzo.

Tous ces animaux ont été abattus à 4 ans et demi faits à l'issue de leur cinquième saison 
des pluies. Ils ont été élevés ensemble du sevrage à la fin de l'expérience et ont passé toute leur vie au même Centre de Recherches.

Ils seront comparés à l'intérieur d'une même année de naissance en raison de la grande importance de ce facteur sur la croissance ultérieure. Le facteur mois de naissance est moins important; les animaux ont été répartis également selon leur mois de naissance à lintérieur de chaque lot, à l'exception du lot A 24 qui serait normalement avantagé puisque ne comportant pas d'animaux nés pendant les deux derniers mois de la saison des naissances.

Les animaux ont été pesés tous les mois et les poids aux âges types obtenus par intrapolations; le relevé du rendement commercial est le rapport du poids de carcasse chaude (avec queue, gras interne, hampe et onglet) au poids vif de départ de la ferme le matin, les abattages étant effectués vers 17 heures.

\section{DEROULEMENT \\ DE L'EXPERIENCE}

L'expérience s'est déroulée normalement. On peut signaler les conditions particulièrement sévères de la saison sèche de 1968. Les animaux de la série $B$ qui étaient au sevrage n'en ont pas été affectés, alors que les animaux de la série A n'ont pratiquement pas grossi entre 1 an et 2 ans. Ce fait donne une idée de la sévérité du milieu où les animaux sont élevés suivant le mode extensif dans cette région.

\section{RESULTATS}

Les résultats sont exploités par une analyse de variance à un facteur contrôlé, selon le modèle suivant :

$\mathrm{x}_{1 \mathrm{\jmath}}=\mathrm{a}_{1}+\Sigma_{\mathrm{i}}$, les nombres de répétitions étant différents (tableau $\mathrm{n}^{*} \mathrm{I}$ ).

TABLEAU $\mathrm{N}^{\circ} \mathrm{I}$

Poids, croissances, rendements.

\begin{tabular}{|c|c|c|c|c|c|c|c|c|c|c|}
\hline & $\mathrm{N}$ & P6 & P12 & P24 & P36 & P48 & P54 & P54-P6 & $\mathrm{P} 54-\mathrm{P} 12$ & $\begin{array}{l}\text { Rendements } \\
(\mathrm{p} .100)\end{array}$ \\
\hline A 12 & 11 & 106,5 & 130,1 & 142,7 & 216,5 & 283,6 & 344,5 & 238,0 & 214,4 & 50,3 \\
\hline A 18 & 9 & 104,6 & 127,1 & 138,2 & 212,9 & 278,2 & 330,7 & 226,1 & 203,6 & 50,7 \\
\hline A 24 & 4 & 122,0 & 151,3 & 149,3 & 213,3 & 271,5 & 325,5 & 203,5 & 174,2 & 50,3 \\
\hline F & $(2 ; 21)$ & 2,957 & 4,866 & 1,021 & 0,086 & 0,497 & 1,204 & 3,665 & 4,424 & 0,468 \\
\hline B 8 & 8 & 103,4 & 131,1 & 181,6 & 240,9 & 327,8 & 381,1 & 277,7 & 250,0 & 51,2 \\
\hline B 12 & 6 & 103,2 & 139,2 & 183,7 & 237,8 & 321,0 & 368,0 & 264,8 & 228,8 & 51,5 \\
\hline $\mathrm{F}$ & $(1 ; 12)$ & 0,002 & 0,816 & 0,045 & 0,115 & $0,28 B$ & 0,693 & 1,031 & 2,160 & 0,184 \\
\hline
\end{tabular}

Série $\mathrm{A}=$ le lot $\mathrm{A} 24$ est au départ significativement plus lourd que les deux autres puisqu'il concernait des animaux présélectionnés sur le poids; cette différence s'estompe avec le temps et est inversée à 4 ans et demi. A cet âge, les animaux castrés à 12 mois sont plus lourds que ceux castrés à 24 mois, sans que ces différences soient significatives. Les croissances sont, par contre, significativement différentes entre les âges de 54 mois et 12 mois, A 12 a grossi de $12 \mathrm{~kg}$ de plus que A 18 et de $40 \mathrm{~kg}$ de plus que $A$ 24. Les rendements ne présentent pas de différences.

Série B : les poids des deux lots sont égaux à 6 mois. A l'âge de 12 mois, les animaux castrés à 8 mois marquent une dépression légère due à la castration. Cette différence s'estompe puis est inversée à 54 mois; B 8 est alors plus lourd que B 12. Les croissances présentent une différence en faveur de B 8 qui n'est pas significative, égale à $13 \mathrm{~kg}$ pour $\mathrm{P}$ 54-P 6 . Les rendements ne présentent pas de différences (tableau $\mathbf{n}^{\circ}$ II).

Série A : quatre rapports anatomiques ont été calculés. L'importance de la longueur scapulo-ischiale (LSI) à la hauteur au garrot (HG), ainsi que celle de la largeur de la tête (IT) par rapport à la longueur (LT), sont plus mar- 
TABLEAU $\mathrm{N}^{\circ} \mathrm{II}$

Mensurations

\begin{tabular}{|l|c|c|c|c|c|c|c|c|c|c|c|}
\hline & PT & HG & LST & LT & II & LH & IH & PT/HG & LSI/HG & IT/LT & IH/LH \\
\cline { 2 - 11 } A 12 & 157,54 & 121,18 & 142,09 & 50,18 & 23,72 & 45,45 & 42,91 & 1,300 & 1,173 & 0,473 & 0,944 \\
\hline A 18 & 156,11 & 121,44 & 138,88 & 50,11 & 22,88 & 44,66 & 42,66 & 1,285 & 1,144 & 0,457 & 0,955 \\
\hline A 24 & 156,50 & 121,00 & 139,25 & 50,00 & 23,00 & 46,00 & 41,00 & 1,294 & 1,151 & 0,460 & 0,891 \\
\hline F & $(2 ; 21)$ & & & & & & & 0,396 & 1,305 & 1,217 & 5,896 \\
\hline B 8 & 167,75 & 123,75 & 149,0 & 49,12 & 22,12 & 46,37 & 46,00 & 1,356 & 1,205 & 0,451 & 0,992 \\
\hline B 12 & 164,83 & 124,33 & 147,66 & 50,50 & 22,50 & 45,83 & 44,33 & 1,326 & 1,187 & 0,446 & 0,967 \\
\hline F & $(1 ; 12)$ & & & & & & & 1,788 & 1,586 & 0,117 & 1,802 \\
\hline
\end{tabular}

quées chez les animaux castrés les plus jeunes, bien que la différence ne soit pas significative. Le rapport de la largeur à la longueur du bassin $(\mathrm{H} / \mathrm{LH})$ montre par contre une différence hautement significative.

Série B : trois rapports présentent une supériorité des animaux castrés les plus jeunes (périmètre thoracique) $\mathrm{PT} / \mathrm{HG}, \mathrm{LSI} / \mathrm{HG}, \mathrm{lH} /$ LH) sans qu'aucune différence significative ne soit observée.

\section{CONCLUSION}

Les conditions matérielles ne nous ont pas permis de disposer de nombres importants d'animaux par lot. Certaines différences n'apparaissent donc pas significatives, bien que proches du seuil de signification.

Certains points sont cependant acquis, à savoir en premier lieu que les croissances pondérales d'animaux élevés selon le mode exten- sif sont fortement influencées par l'âge de la castration, les animaux castrés les plus jeunes ayant une croissance ultérieure supérieure, ceci dans les limites d'âges de castration pratiquées, c'est-à-dire entre 8 mois et 24 mois. Des essais complémentaires devront porter sur la castration à des âges inférieurs à 8 mois. Les rendements en carcasses nont, par contre, pas présenté de différences. Pour les rapports anatomiques, l'indice de largeur du bassin semble le plus concerné par l'âge de la castration, dans les deux séries. Les autres rapports ne présentent pas de différences statistiquement significatives.

De ces observations, il résulte que l'éleveur de Zébus destinés à la boucherie en tant que bœuf d'herbe a tout intérêt à les castrer au cours de leur première année. Il serait très profitable pour la rentabilité de l'élevage malgache que cette technique soit vulgarisée, opération qui serait à la fois peu coûteuse, puisque la castration est pratiquée par les éleveurs euxmêmes, et ne devrait pas présenter de difficultés psychologiques majeures.

\section{SUMMARY}

\section{Incidence of the age at the castration on zebu cattle growth and dressing percentage in Madagascar}

In Madagascar, most of the breeders practise extensive beef production with the Zebu malagasy breed. They castrate their animals between 2 and 4 years old.

The authors have studied the incidence of the age at the castration on the following growth, when castrating the animals at $8,12,18$ and 24 months old; all these steers have been slaughtered at 4,5 years old, after a traditional management. In this test, decreasing age at castration increases the following growth rate, has no effect on the carcass yield and increases the width-length ratio of the hip. 


\section{RESUMEN}

Influencia de la edad en el momento de la castración sobre el desarrollo y el rendimiento del cebú, en Madagascar

En Madagascar, la casi totalidad de los ganaderos practican una ganaderia extensiva de animales de raza Cebú malgacho para la producción de carne, con alimentación sin aditivos.

Se efectua la castración de los animales entre 2 y 4 años de edad. Los autores estudiaron la influencia de la edad en el momento de la castración sobre el desarrollo ulterior, castrando los animales a 8 meses, 12 meses, 18 meses y 24 meses; se mataron todos los animales a 4 años y media de edad después de una ganaderia según el modo tradicional. En esta experiencia, la baja de la edad de la castración aumenta el crecimiento ponderal global, no tiene ninguna influencia sobre el rendi miento de la canal y aumenta la relación anchura sobre longitud de lá pelvis.

\section{BIBLIOGRAPHIE}

1. BAILEY (C. B.) et HIRONAKA (R.). Growth and carcass characteristics of bulls, steers and partial castrates kept on range for the first year of life and then fattened. Can. J. anim. Sci., 1969, 49 : 37-44.

2. BRANNANG (E.). Studies on monozygous cattle twins XVIII. The effect of castration and age of castration on the growth rate, feed conversion and carcass traits of Swedish Red and White cattle. I. Anim. breed, Abstr., 1969, 37 (4) : 5750. (Abstr. n० 3297).

3. GEAY (Y.) et MALTERRE (C.). Influence de la castration et de la nature des glucides de la ration sur la croissance et la qualité des carcasses de bovins abattus à 24 mois. Ann. Zootech., 1971, 20 (2) : 251-257.

4. GILIBERT (J.). Valeurs bouchères du zébu à
Madagascar. Rev. Elev. Méd. vét. Pays trop., 1971, 24 (3) : 445-465.

5. HARTE (F. J.). The production of beef from young bulls, III. Ir. J. agric. Res., 1969, 8: 293305.

6. KING (G. T.) et CARPENTER (Z. L.). Cutability of bull, steer and heifer carcasses. Beef cartle Res. Tex., 1967, 38-41.

7. MIETH (K.) et BERG (F.). Fattening of young bulls at pasture following different methods of castration and sterilisation. Mh. Vet. Med., 1969, 24: 168-171.

8. La technique de castration des jeunes bovins de boucherie. Rev. Elev., 1967, 22 (11): 35.

9. WATSON (M. J.). The effects of castration on the growth and meat quality of grazing cattle. Aust. J. exp. Agric. anim. Husb., 1969, 9 : 164-171. 